\title{
Modification of Disney trap for capture of sand flies (Diptera: Psychodidae: Phlebotominae)
}

\author{
Maria Elizabeth C Dorval, Tulia Peixoto Alves, Alessandra Gutierrez de Oliveira, \\ Reginaldo Peçanha Brazil*/+, Eunice Aparecida Bianchi Galati**, \\ Rivaldo Venâncio da Cunha***
}

\begin{abstract}
Departamento de Patologia ***Departamento de Clínica Médica, Faculdade de Medicina, Universidade Federal de Mato Grosso do Sul, Campo Grande, MS, Brasil *Departamento de Bioquímica e Biologia Molecular, Instituto Oswaldo Cruz-Fiocruz, Av. Brasil 4365, 21045-900 Rio de Janeiro, RJ,Brasil **Departamento de Epidemiologia, Faculdade de Saúde Pública, USP, São Paulo, SP, Brasil

This paper describes the modifications made to the original model of the Disney trap, with a view to easier handling of the same, greater practicability in the collection of sand flies, protection of the animal bait and durability of the trap in the field.
\end{abstract}

Key words: Psychodidae - Phlebotominae - Disney trap - animal bait

Phlebotomine sand flies are diptera of great significance in public health, because, beyond being vectors of protozoa of the genus Leishmania, etiologic agents of leishmaniasis, they are also incriminated as vectors of arboviroses and bartonelosis (Miles et al. 1983, Tesh 1988, Killick-Kendrick 1990, Lainson \& Rangel 2005).

Different methods of capture are necessary for the studies of sand fly-parasite relationships, vector behavior, taxonomy and ecology (Alexander 2000).

Among the traps that use animal as baits, the Disney's (Disney 1966) consists of a metal tray with a cage, holding the chosen bait, in the middle. Castor oil, for the capture of the insects attracted to the animal bait, is sprayed onto the tray. The insects, before or after contact with the bait, stick to the oil when they land on the surface of the tray.

At the beginning of the project on the sand fly fauna in a cutaneous leishmaniasis endemic area, Disney traps (as described by Maroli et al. 1997) were used to capture those species of low flight and zoophilic habits. However, the traps installed in forested areas were damaged by predators and did not thus offer sufficient security to the animal used as bait (normally a hamster, Mesocricetus auratus).

To solve this problem, the trap was modified as follows. The cage was placed between two galvanized zinc sheets $(50 \times 50 \mathrm{~cm})$, with aluminum corners, which formed the roof and floor of the same. These sheets were placed so as to slide in such away as to facilitate cleaning

Financial support: Fundect/Decit/Protocol 00645-03, UFMS

+ Corresponding author: rpbrazil@ioc.fiocruz.br

Received 26 October 2006

Accepted 18 September 2007 and the visualization and collection of the insects captured. Both of the sheets were sprayed with castor oil. The feet of the trap were removable to facilitate its installation (Figs 1, 2). In this study, the animals were housed in plastic boxes about $30 \mathrm{~cm}$ from the ground covered with an iron mesh, individually in accordance with the population density recommendations of IIar (1996), and on beds of wood (Pinnus sp.) shavings, being fed on Nuvilab CR-1 commercial food (Nuvital, Curitiba, PR, Brazil) and receiving water ad libitum. This type of box protected the animal better and prevented food residues and excrement from falling onto the lower sheet, damaging the insects, and making the visualization and withdrawal of the flies more difficult. The boxes were cleaned and the food replenished every week. An iron cage was constructed for the greater safety of the bait and was locked so as to prevent the traps being disturbed and the animals being removed or attacked (Fig. 3 ). As a result only two of the 36 animals used as sentinels died.

With the modified trap 1,191 sand flies (representing $59.6 \%$ of the total specimens collected) were caught in it. The remaining percentage was caught in Shannon and automatic light traps (unpublished observations). This may be explained by the fact that the modified Disney trap captured insects continuously whereas the others were only installed periodically and at night. Beyond having been effective, the modified trap showed selectivity in relation to the capture of certain species (Table). Evandromyia bourrouli (Barretto \& Coutinho, 1941) contributed $57.3 \%$ of the total of sand flies captured on this project and it was captured exclusively with this trap.

Bichromomyia flaviscutellata (Mangabeira, 1942), known as a rodentophilic species, although it was also collected by the other two methods, accounted for $94.1 \%$ of the specimens captured in the modified Disney trap, $81.7 \%$ of them being females.

The continuous use of this type of trap, with the periodical removal of the zinc sheets, makes the study of the nictemeral behavior of the sand fly species possible. 


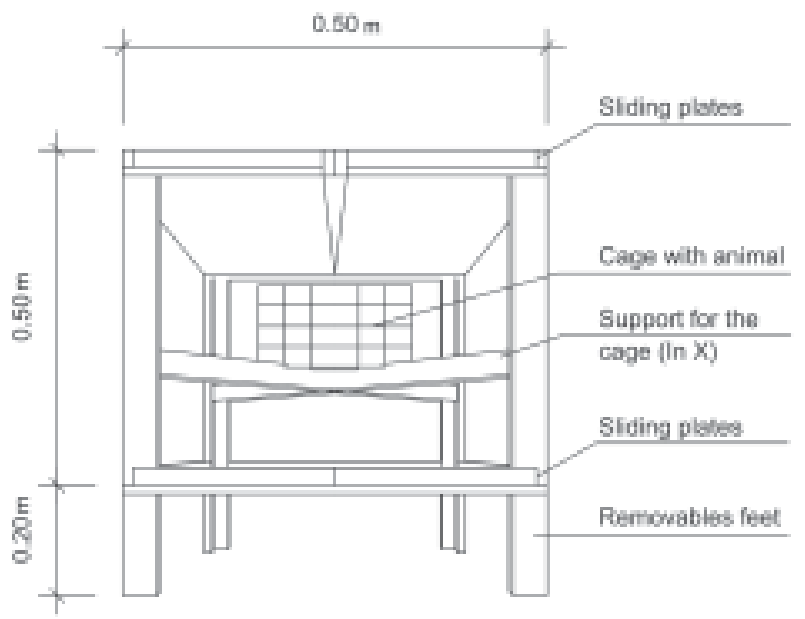

Fig. 1: schematic drawing of modified Disney trap.
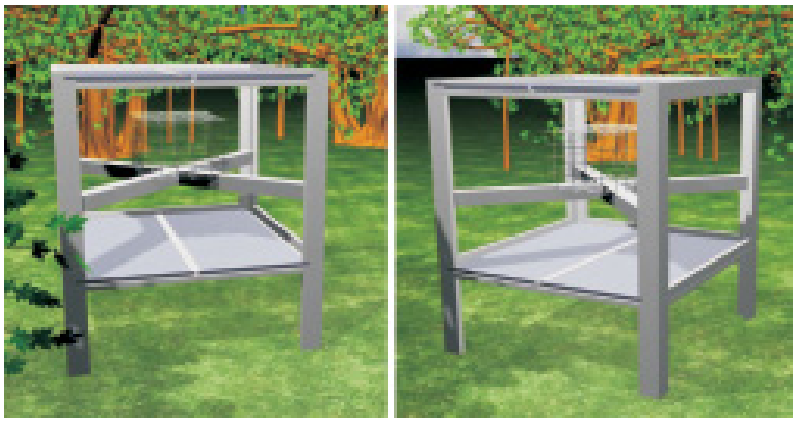

Fig. 2: artistic drawing of modified Disney trap.

\section{ACKNOWLEDGEMENTS}

To Mr Jorge da Silva Oliveira for the preparation of the drawings.

\section{REFERENCES}

Alexander B 2000. Sampling methods for phlebotomine sandflies. Med Vet Entomol 14: 109-122.

Disney RHL 1966. A trap for phlebotomine sand flies attracted to rats. Bull Entomol Res 56: 445-451.

ILAR-Institute of Laboratory Animal Resources. National Research Council 1966. Guide for Care and Use of Laboratory Animals, National Academic Press, 126 pp.

Killick-Kendrick R 1990. Phlebotomine vectors of the leishmaniasis: a review. Med Vet Entomol 4: 1-24.

Lainson R, Rangel EF 2005. Lutzomyia longipalpis and ecoepidemiology of American visceral leishmaniais, with par-
TABLE

Relative abundance by species of sand flies captured with modified Disney trap in the municipality of Bela Vista, Mato Grosso do Sul, Brazil, from May 2004 to January 2006

\begin{tabular}{lccrr}
\hline Species & Males & Females & Total & $\%$ \\
\hline Bichromomyia flaviscutellata & 28 & 465 & 493 & 41.40 \\
Brumptomyia avellari & 4 & 0 & 4 & 0.34 \\
Brumptomyia brumpti & 3 & 1 & 4 & 0.34 \\
Evandromyia bourrouli & 582 & 100 & 682 & 57.26 \\
Evandromyia lenti & 1 & 1 & 2 & 0.17 \\
Lutzomyia longipalpis & 1 & 1 & 2 & 0.17 \\
Psathyromyia campograndensis & 0 & 1 & 1 & 0.08 \\
Psathyromyia punctigeniculata & 0 & 1 & 1 & 0.08 \\
Psathyromyia shannoni & 1 & 0 & 1 & 0.08 \\
Sciopemyia sordelli & 1 & 0 & 1 & 0.08 \\
\hline Total & 622 & 569 & 1191 & 100 \\
\hline
\end{tabular}

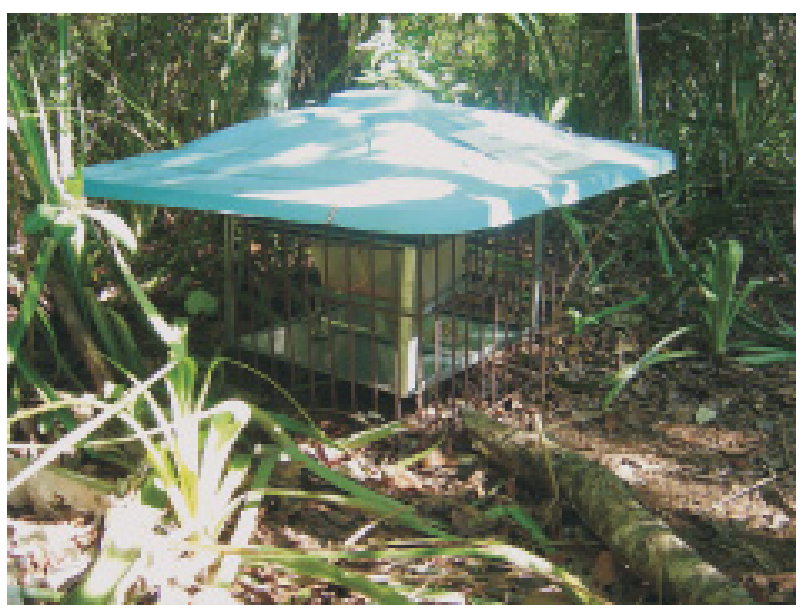

Fig. 3: modified Disney trap, installed in a forested area of the $10^{\circ}$ Regimento de Cavalaria Mecanizado, Bela Vista, MS, Brazil.

ticular reference to Brazil: a review. Mem Inst Oswldo Cruz 100: 811-827.

Miles MA, Arias JR, Valente SAS, Naiff RD, Souza AA, Póvoa MM, Lima JAN, Cedillos RA 1983. Vertebrate hosts and vectors of Trypanosoma rangeli in the Amazon basin of Brazil. Am J Trop Med Hyg 32: 1251-1259.

Maroli M, Feliciangeli MD, Arias J 1997. Métodos de Captura, Conservacion y Montaje de los Flebótomos (Diptera: Psychodidae), OPS/OMS, OPS/HCP/HCT/95/97, 72 pp.

Tesh RB 1988. The genus Phlebovirus and its vectors. Ann Rev Entomol 33: 169-181. 